\title{
Computer Time Minimizing for the Circuit Optimization
}

\author{
ALEXANDER ZEMLIAK \\ Department of Physics and Mathematics \\ Autonomous University of Puebla \\ Av. San Claudio y Rio Verde, Puebla, 72570 \\ MEXICO \\ Institute of Physics and Technology \\ National Technical University of Ukraine \\ UKRAINE \\ azemliak@yahoo.com
}

\begin{abstract}
The solution of a problem of analogue circuit optimization is mathematically defined as a controllable dynamic system. In this context the minimization of the processor time of designing can be formulated as a problem of time minimization for transitional process of dynamic system. A special control vector that changes the internal structure of the equations of optimization procedure serves as a principal tool for searching the best strategies with the minimal CPU time. In this case a well-known maximum principle of Pontryagin is the best theoretical approach for finding of the optimum structure of control vector. Practical approach for realization of the maximum principle is based on the analysis of behaviour of a Hamiltonian for various strategies of optimization. It is shown that in spite of the fact that the problem of optimization is formulated as a nonlinear task, and the maximum principle in this case isn't a sufficient condition for obtaining a minimum of the functional, it is possible to obtain the decision in the form of local minima. The relative acceleration of the CPU time for the best strategy found by means of maximum principle compared with the traditional approach is equal two to three orders of magnitude.
\end{abstract}

Key-Words: - Analog circuits optimization, optimal strategy, theory of control, maximum principle, acceleration effect.

Received: October 7, 2019. Revised: March 13, 2020. Accepted: April 23, 2020. Published: April 30, 2020.

\section{Introduction}

The problem of reducing processor time spent on the optimization of electronic circuits is one of the important problems associated with improving the quality of design. Some works devoted to this problem are devoted to how to reduce the number of operations in solving two main problems: circuit analysis and numerical optimization. Solving these problems gives us a significant reduction in CPU time. Methods that can be used in the analysis of complex systems are being improved. Some ideas regarding the use of the sparse matrix method [1-2] and decomposition methods [3] are used to reduce the analysis time of circuits. Other alternative methods such as homotopy methods [4] were successfully applied for circuit analysis too.

Some methods of optimization were developed for circuit designing, timing, and area optimization [5-6]. However, classical deterministic optimization algorithms may have a number of drawbacks: they may require that a good initial point be selected in the parameter space, they may reach an unsatisfactory local minimum, and they require that the cost function be continuous and differentiable.
To overcome these issues, special methods were applied to determine the initial point of the process by centering [7], or by applying geometric programming methods [8].

Other formulation of the circuit optimization problem was developed at a heuristic level some decades ago [9]. This approach ignored Kirchhoff's laws for all the circuit or part of it. The practical aspects of this idea were developed for the optimization of microwave circuits [10] and for the synthesis of high-performance analog circuits [11] in case where all the equations of the circuit model were not solved during the total optimization process.

The new formulation of the problem of circuit optimization is formulated in terms of the theory of optimal control [12-13]. In this case the process of circuit optimization was generalized and defined as the dynamic controllable system. A basic element is the control vector that changes the structure of the equations of system of optimization process. Thus there is a set of the strategies of optimization that have different number of operations and different processor time. Introduction of the function of 
Lyapunov of the optimization process [14] allows to compare various strategies of optimization and to choose the best of them having the minimum processor time. At the same time, the solution to the problem of finding the optimal strategy and the corresponding optimal trajectory can be found within the framework of the Pontryagin maximum principle [15].

The main complexity of application of the maximum principle consists of the search of initial values for auxiliary variables at the solution of the conjugate system of equations. Application of the maximum principle in case of linear dynamic systems is based on the creation of an iterative process [16-17]. In case of nonlinear systems, the convergence of this process is not guaranteed. However, application of the additional approximating procedures [18-19] allows constructing sequence of the solutions converging to a limit under certain conditions.

Section 2 gives the formulation of the circuit optimization process based on the methods of control theory using a control vector. Section 3 gives an example of the application of the maximum principle for optimizing the simplest nonlinear circuit. It is shown that analysis of the Hamiltonian behaviour allows one to obtain the exact structure of a control vector that minimizes processor time.

\section{Problem Formulation}

We define the optimization process for analog circuit as the problem of minimization of the generalized cost function $F(X, U)$ by the equation (1) with the constraints (2):

$$
\begin{gathered}
X^{s+1}=X^{s}+t_{s} \cdot H^{s} \\
\left(1-u_{j}\right) g_{j}(X)=0, j=1,2, \ldots, M
\end{gathered}
$$

where $X \in R^{N}, X=\left(X^{\prime}, X^{\prime \prime}\right), X^{\prime} \in R^{K}$ is the vector of the independent variables and the vector $X^{\prime \prime} \in R^{M}$ is the vector of dependent variables $(N=K+M), s$ is the iterations number, $t_{s}$ is the iteration parameter, $t_{s} \in R^{1}, g_{j}(X)$ presents the equation $j$ of the circuit's model, $H \equiv H(X, U)$ is the direction of the generalized cost function $F(X, U)$ decreasing, $U$ is the vector of the special control functions $U=\left(u_{1}, u_{2}, \ldots, u_{m}\right)$, where $u_{j} \in \Omega$; $\Omega=\{0 ; 1\}$. The generalized cost function $F(X, U)$ is defined as:

$$
F(X, U)=C(X)+\psi(X, U),
$$

where $C(X)$ is the non-negative cost function of the designing process, and $\psi(X, U)$ is the additional penalty function:

$$
\psi(X, U)=\frac{1}{\varepsilon} \sum_{j=1}^{M} u_{j} \cdot g_{j}^{2}(X) .
$$

By means of this formulation we redistribute the computer time expense between the solution of problem (2) and the optimization procedure (1) for the function $F(X, U)$. The control vector $U$ is the main tool for the redistribution process in this case. The problem of search of the optimal design strategy with a minimal CPU time is formulated as the typical problem for the functional minimization of the control theory. The functional that needs to minimize is the total CPU time $T$ of the design process. This functional depends on the operations number and on the strategy of designing that has been realized. The main difficulty of this definition is unknown optimal dependencies of all control functions $u_{j}$.

The Eq. (1) can be replaced by the differential equation in continuous form using the next formula:

$$
\frac{d x_{i}}{d t}=f_{i}(X, U), \quad i=1,2, \ldots, N
$$

The equations (2), (3), (4) and (5) compose the continuous form of the design process. The functions of the right hand part of the system (5) can be determined for example for the gradient method by means of the next expression:

$$
\begin{aligned}
& f_{i}(X, U)=-\frac{\delta}{\delta x_{i}} F(X, U), \quad i=1,2, \ldots, K, \\
& f_{i}(X, U)=-u_{i-K} \frac{\delta}{\delta x_{i}} F(X, U)+\frac{\left(1-u_{i-K}\right)}{t_{s}}\left\{-x_{i}^{s}+\eta_{i}(X)\right\} \\
& i=K+1, K+2, \ldots, N,
\end{aligned}
$$

where the operator $\delta / \delta x_{i}$ hear and below means $\frac{\delta}{\delta x_{i}} \varphi(X)=\frac{\partial \varphi(X)}{\partial x_{i}}+\sum_{p=K+1}^{K+M} \frac{\partial \varphi(X)}{\partial x_{p}} \frac{\partial x_{p}}{\partial x_{i}}$, $x_{i}^{s}$ is equal to $x_{i}(t-d t) ; \eta_{i}(X)$ is the implicit function $\left(x_{i}=\eta_{i}(X)\right)$ that is determined by system (2).

The control variables $u_{j}$ have the time 
dependency in general case. The equation number $j$ is removed from (2) and the dependent variable $x_{K+j}$ is transformed to the independent when $u_{j}=1$.

This independent parameter is defined by the formulas (5), $\left(6^{\prime}\right)$. In this case there is no difference between formulas (6) and (6'). The transformation of the vectors $X^{\prime}$ and $X^{\prime \prime}$ can be done at any time moment.

We need to find the optimal behavior of the control functions $u_{j}$ during the optimization process to minimize the total computer time of designing. The more adequate method for solution of this problem is a maximum principle of Pontryagin.

\section{Maximum Principle Application}

The main complexity of application of the maximum principle consists of the search of initial values for auxiliary variables at the solution of the conjugate system of equations. Application of the maximum principle for the linear dynamic systems is based on creation of iterative process [16].

In case of nonlinear systems the convergence of this process isn't guaranteed, however application of the additional approximating procedures [17-19] allows constructing sequence of the solutions meeting to a limit under certain conditions. In the present work the possibility of application of the maximum principle for creation of the optimal control vector and the optimal trajectory of optimization process corresponding to it is investigated. The example of optimization of the simplest nonlinear circuit for which the analytical solution of the task was obtained is investigated. We will consider a nonlinear circuit of a voltage divider in Fig. 1.

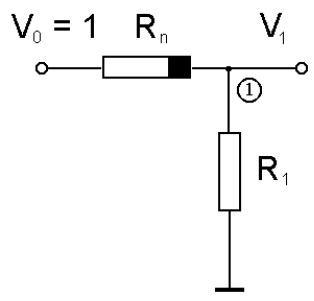

Fig.1 Simplest nonlinear circuit of voltage divider

Let us consider that the nonlinear element has the following dependence:

$$
R_{n}=a+b\left(V_{1}-V_{0}\right)
$$

where $a>0, b>0, a>b, V_{0}$ and $V_{1}$ the voltages on an entrance and an exit of circuit.
We will consider that $V_{0}$ is equal 1 . We will define the variables $x_{1}, x_{2} . x_{1}=R, x_{2}=V_{1}$. Thus the vector of phase variables $X \in R^{2}$. In this case the formula (7) can be replaced with the following expression:

$$
R_{n}=a+b\left(x_{2}-1\right)
$$

We can present the equation of a circuit in the form:

$$
g_{1}\left(x_{1}, x_{2}\right) \equiv x_{2}\left[x_{1}+a+b\left(x_{2}-1\right)\right]-x_{1}=0
$$

The circuit optimization is formulated as a problem of obtaining at the exit of a circuit of the defined voltage $w$. We will determine the cost function of the optimization process by a formula:

$$
C(X)=\left(x_{2}-w\right)^{2} .
$$

In this case the problem of circuit optimization is converted to minimization of the cost function $C(X)$. Following theoretical basis, that were developed in [12], we formulate the problem for circuit optimization as a task of search of the optimization strategy with a minimum possible CPU time. For this purpose we define the functional, which is subject to minimization, by the following expression:

$$
J=\int_{0}^{T} f_{0}(X) d t
$$

where $f_{0}(X)$ the function which is conditionally determining density of number of arithmetic operations in unit of time of $t$. In that case, the integral (5) defines total number of operations necessary for circuit optimization and is proportional to the total CPU time.

The structure of function $f_{0}(X)$ can't be defined, however we can compute CPU time, using possibilities of the compiler. We will identify further the integral (11) with CPU time and therefore the problem of minimization of CPU time corresponds to a problem of minimization of the integral (11).

According to [12] we introduce the control vector $U$ that consists of only one component $u(t)$ for the reviewed example. This component has one of two possible values: 0 or 1 . The control vector allows to generalize the circuit optimization process and to define a set of the optimization strategies differing in operations number and CPU time. The 
generalized cost function can be defined in this case by means of the formula:

$$
F(X)=C(X)+\varphi(X),
$$

where $\varphi(X)$ is an additional penalty function, which can be determined, for example, by the following formula:

$$
\varphi(X)=\sum_{j=1}^{M} u_{j} \cdot g_{j}^{2}(X),
$$

where $M$ is the number of nodes of the circuit. In our case $M=1$. The process of circuit optimization thus can be described by the system (14) with restrictions (15):

$$
\begin{gathered}
\frac{d x_{i}}{d t}=f_{i}\left(x_{1}, x_{2}, u\right), \quad i=1,2, \\
(1-u) g_{1}\left(x_{1}, x_{2}\right)=0,
\end{gathered}
$$

where functions $f_{i}\left(x_{1}, x_{2}, u\right)$ are defined by a concrete numerical method of optimization. When using a gradient method these functions are defined by the following formulas:

$$
f_{i}\left(x_{1}, x_{2}, u\right)=-\frac{\delta}{\delta x_{i}} F(X), \quad i=1,2 .
$$

The value $u(t)=0$ corresponds to the traditional strategy of optimization (TSO). In this case in system (14) there is only one equation for the independent $x_{1}$ variable while the variable $x_{2}$ is defined from the equation (15). The value $u(t)=1$ corresponds to the modified traditional strategy of optimization (MTSO) when both $x_{1}$ and $x_{2}$ variables are independent. In this case the system (14) includes two equations for the independent variables $x_{1}$ и $x_{2}$, and the equation (15) disappears. Change of the value of function $u(t)$ with 0 on 1 and back can be made at any moment, and generates a set of various strategies of optimization. Two main strategies of structural basis can be defined by means of the next two approaches.

1) TSO, $u=0$. The equations (14)-(16) are replaced with the following equations:

$$
\begin{aligned}
& \frac{d x_{1}}{d t}=-\frac{\partial C}{d x_{2}} \frac{d x_{2}}{d x_{1}} \\
& \frac{d x_{2}\left(x_{1}, t\right)}{d t}=\frac{\partial x_{2}}{\partial x_{1}} \frac{d x_{1}}{d t}
\end{aligned}
$$

where the derivative $d x_{2} / d x_{1}$ is defined from the equation (9), and $\frac{d x_{2}}{d x_{1}}=\frac{1}{2 b}\left[-1+\frac{x_{1}+c+2 b}{\sqrt{\left(x_{1}+c\right)^{2}+4 b x_{1}}}\right]$, $c=a-b$.

2) MTSO, $u=1$. The equations (14) are transformed to the next one:

$$
\frac{d x_{i}}{d t}=-\frac{\delta}{\delta x_{i}}\left[C(X)+g_{1}^{2}(X)\right], \quad i=1,2 .
$$

In general case the right hand parts of the equations (14) can be presented in the form:

$$
\begin{aligned}
& f_{1}\left(x_{1}, x_{2}, u\right)=(1-u) \cdot f_{11}\left(x_{1}, x_{2}\right)+u \cdot f_{12}\left(x_{1}, x_{2}\right), \\
& f_{2}\left(x_{1}, x_{2}, u\right)=(1-u) \cdot f_{21}\left(x_{1}, x_{2}\right)+u \cdot f_{22}\left(x_{1}, x_{2}\right),
\end{aligned}
$$

where the functions $f_{i j}\left(x_{1}, x_{2}\right)$ are determined by the following formulas:

$$
\begin{aligned}
f_{11}\left(x_{1}, x_{2}\right)= & \frac{\left(w-x_{2}\right)}{b}\left[-1+\frac{x_{1}+c+2 b}{\sqrt{\left(x_{1}+c\right)^{2}+4 b x_{1}}}\right] \\
f_{12}\left(x_{1}, x_{2}\right)= & -2\left(x_{2}-1\right)\left\{\left(x_{2}-1\right) x_{1}+\left[a+b\left(x_{2}-1\right)\right] x_{2}\right\} \\
f_{21}\left(x_{1}, x_{2}\right)= & \frac{\left(w-x_{2}\right)}{2 b^{2}}\left[-1+\frac{x_{1}+a+b}{\sqrt{\left(x_{1}+c\right)^{2}+4 b x_{1}}}\right]^{2} \\
f_{22}\left(x_{1}, x_{2}\right)= & -2\left\{\left(x_{2}-w\right)+\left(c+x_{1}+2 b x_{2}\right)\right. \\
& \left.\cdot\left[\left(x_{2}-1\right) x_{1}+a x_{2}+b\left(x_{2}-1\right) x_{2}\right]\right\}
\end{aligned}
$$

According to methodology of the maximum principle, the system of the conjugate equations for additional variables $\psi_{1}, \psi_{2}$ has the next form:

$$
\begin{aligned}
& \frac{d \psi_{1}}{d t}=-\frac{\partial f_{1}\left(x_{1}, x_{2}, u\right)}{\partial x_{1}} \cdot \psi_{1}-\frac{\partial f_{2}\left(x_{1}, x_{2}, u\right)}{\partial x_{1}} \cdot \psi_{2}, \\
& \frac{d \psi_{2}}{d t}=-\frac{\partial f_{1}\left(x_{1}, x_{2}, u\right)}{\partial x_{2}} \cdot \psi_{1}-\frac{\partial f_{2}\left(x_{1}, x_{2}, u\right)}{\partial x_{2}} \cdot \psi_{2},
\end{aligned}
$$

where the partial derivatives of functions $f_{i}\left(x_{1}, x_{2}, u\right), i=1,2$ can be calculated by formulas (20), (23). 


$$
\begin{aligned}
\frac{\partial f_{11}\left(x_{1}, x_{2}\right)}{\partial x_{1}}= & \frac{\left(x_{2}-w\right) 4 a}{\left[\left(x_{1}+c\right)^{2}+4 b x_{1}\right]^{3 / 2}} \\
\frac{\partial f_{12}\left(x_{1}, x_{2}\right)}{\partial x_{1}}= & -2\left(x_{2}-1\right)^{2} \\
\frac{\partial f_{21}\left(x_{1}, x_{2}\right)}{\partial x_{1}}= & -\frac{\left(w-x_{2}\right)}{b}\left[-1+\frac{x_{1}+a+b}{\sqrt{\left(x_{1}+c\right)^{2}+4 b x_{1}}}\right] \\
& \cdot \frac{4 a}{\left[\left(x_{1}+c\right)^{2}+4 b x_{1}\right]^{3 / 2}}
\end{aligned}
$$$$
\begin{aligned}
\frac{\partial f_{22}\left(x_{1}, x_{2}\right)}{\partial x_{1}}=- & -2\left\{\left(x_{2}-1\right) x_{1}+\left[a+b\left(x_{2}-1\right)\right] x_{2}\right. \\
& \left.+\left(c+x_{1}+2 b x_{2}\right)\left(x_{2}-1\right)\right\}
\end{aligned}
$$$$
\frac{\partial f_{11}\left(x_{1}, x_{2}\right)}{\partial x_{2}}=-\frac{1}{b}\left[-1+\frac{x_{1}+a+b}{\sqrt{\left(x_{1}+c\right)^{2}+4 b x_{1}}}\right]
$$$$
\frac{\partial f_{12}\left(x_{1}, x_{2}\right)}{\partial x_{2}}=-4\left(x_{2}-1\right) x_{1}-2\left[a+b\left(x_{2}-1\right)\right] x_{2}
$$$$
-2\left(x_{2}-1\right)\left[b x_{2}+a+b\left(x_{2}-1\right)\right]
$$$$
\frac{\partial f_{21}\left(x_{1}, x_{2}\right)}{\partial x_{2}}=-\frac{1}{2 b^{2}}\left[-1+\frac{x_{1}+a+b}{\sqrt{\left(x_{1}+c\right)^{2}+4 b x_{1}}}\right]^{2}
$$$$
\begin{aligned}
\frac{\partial f_{22}\left(x_{1}, x_{2}\right)}{\partial x_{2}}=- & 2\left\{1+2 b\left[\left(x_{2}-1\right) x_{1}+b x_{2}^{2}+c x_{2}\right]\right. \\
+ & \left.\left(c+x_{1}+2 b x_{2}\right)^{2}\right\}
\end{aligned}
$$

The Hamiltonian is expressed by the following formula:

$$
H=\psi_{1} \cdot f_{1}\left(x_{1}, x_{2}, u\right)+\psi_{2} \cdot f_{2}\left(x_{1}, x_{2}, u\right)
$$

Substituting (14) in (18) and doing identical transformations we obtain the following expression for a Hamiltonian:

$$
\begin{aligned}
H= & \psi_{1} \cdot f_{11}\left(x_{1}, x_{2}\right)+\psi_{2} \cdot f_{21}\left(x_{1}, x_{2}\right) \\
& +u \cdot \Phi\left(x_{1}, x_{2}, \psi_{1}, \psi_{2}\right)
\end{aligned}
$$

where

$$
\begin{aligned}
\Phi\left(x_{1}, x_{2}, \psi_{1}, \psi_{2}\right) & =\psi_{1} \cdot\left[f_{12}\left(x_{1}, x_{2}\right)-f_{11}\left(x_{1}, x_{2}\right)\right] \\
& +\psi_{2} \cdot\left[f_{22}\left(x_{1}, x_{2}\right)-f_{21}\left(x_{1}, x_{2}\right)\right]
\end{aligned} .
$$

According to the maximum principle, we obtain the next main condition for the control function $u$ :

$$
u= \begin{cases}0, & \Phi<0 \\ 1, & \Phi>0\end{cases}
$$

The behavior of the control function $u(t)$ that corresponds to the maximum principle is defined also by behavior of functions $\psi_{1}(t)$ and $\psi_{2}(t)$, which are computed from the equations (22). At the same time the solution of the equations (22) depends on initial values $\psi_{10}$ and $\psi_{20}$, which are defined within the precision of common multiplier. One of these constants can be taken arbitrary. Let us define the constant $\psi_{10}=-1$. The value of $\psi_{20}$ that corresponds to the adequate solution of a task in the conditions of the maximum principle can be obtained by iterative procedure. We use iterative procedure on the basis of the Newton method that provides the solution for the minimum time.

The analysis of optimization process for a similar example which is carried out in work [17] showed that the TSO $(u=0)$ is optimal one when initial values of variables $x_{1}$ and $x_{2},\left(x_{10}, x_{20}\right)$ are positive. At the same time the negative initial values of the variable $x_{2}$ leads to significantly other results. In case of negative initial values of variable $x_{20}$, emergence of effect of acceleration of the process of circuit optimization is possible. This effect accelerates the optimization process in some times. It is interesting to check, whether it is possible to obtain similar result on the basis of maximum principle.

Fig. 2 shows the trajectory of the process of circuit optimization in phase space of two variables $x_{1}, x_{2}$, corresponding to the initial point $\left(x_{10}=1\right.$, $x_{20}=1$ ) that was obtained with a main condition of the maximum principle (26).

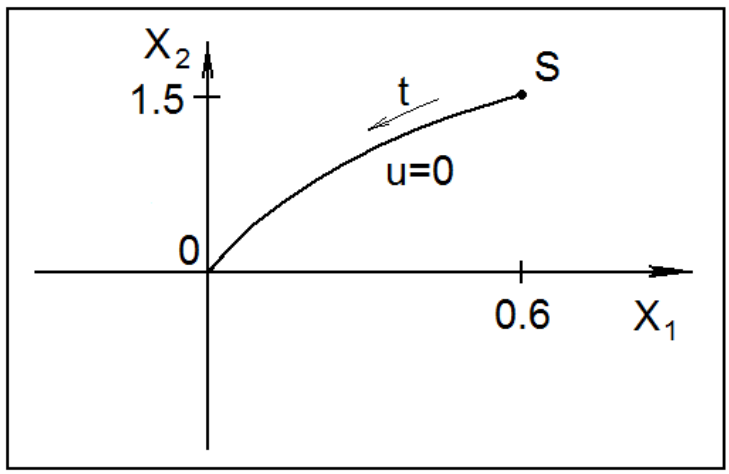

Fig. 2 Trajectories of optimization process in phase space for initial point $x_{10}=1, x_{20}=1$ 
In this case the optimum trajectory corresponds to TSO and the constant value $u=0$. Thus the number of iterations is equal to 3719 and time of the CPU is equal to $20.45 \mathrm{msec}$. Changing of the initial point of $\mathrm{S}$ at any other positive values of coordinates $x_{1}, x_{2}$, doesn't lead to change of a trajectory. However the negative value of coordinate $x_{2}$ leads to essential change of the solution. The trajectories of process of circuit optimization corresponding to the initial point $\left(x_{10}=1, x_{20}=-1\right)$ are shown in Fig. 3.

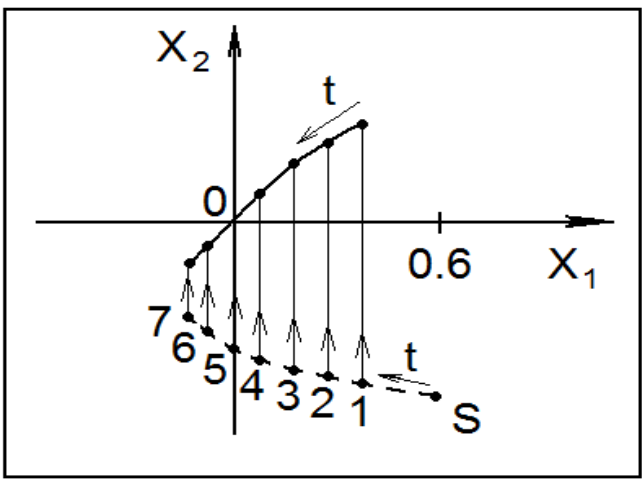

Fig. 3 trajectories of optimization process in phase space for initial point $x_{10}=1, x_{20}=-1$

The structure of function $u(t)$ that was obtained automatically and corresponds to a condition of the maximum principle (26) has one point of a rupture that corresponds to switching from the trajectory corresponding to MTSO ( $u=1$, a dotted curve) on trajectory corresponding to TSO $(u=0$, a continuous curve). Coordinates of a point of switching of $t_{\mathrm{sw}}$ depend on value of $\psi_{20}$. The data corresponding to points 1, 2, 3, 4, 5, 6 and 7 in Fig. 3 are presented in Table 1.

Table 1. Data for different initial value

\begin{tabular}{|r|r|r|r|}
\hline $\mathrm{N}$ & $\psi_{20}$ & \multicolumn{1}{l|}{$\begin{array}{l}\text { lterations } \\
\text { number }\end{array}$} & $\begin{array}{l}\text { Time } \\
(\mathrm{msec})\end{array}$ \\
\hline 1 & 40.00 & 3568 & 19.620 \\
\hline 2 & 30.00 & 3383 & 18.613 \\
\hline 3 & 20.00 & 2790 & 15.351 \\
\hline 4 & 16.00 & 1810 & 9.962 \\
\hline 5 & 14.35 & 277 & 1.520 \\
\hline 6 & 10.00 & 1152 & 6.310 \\
\hline 7 & 2.00 & 1887 & 10.781 \\
\hline
\end{tabular}

Change of the value of $\psi_{20}$ from 40.0 till 14.35 leads to reduction of iterations number and CPU time from $19.62 \mathrm{msec}$ to $1.520 \mathrm{msec}$, but the CPU time is increasing later on. That is visible also in Fig. 4, where the dependence of CPU time of the solution of a task from initial value $\psi_{20}$ is shown.

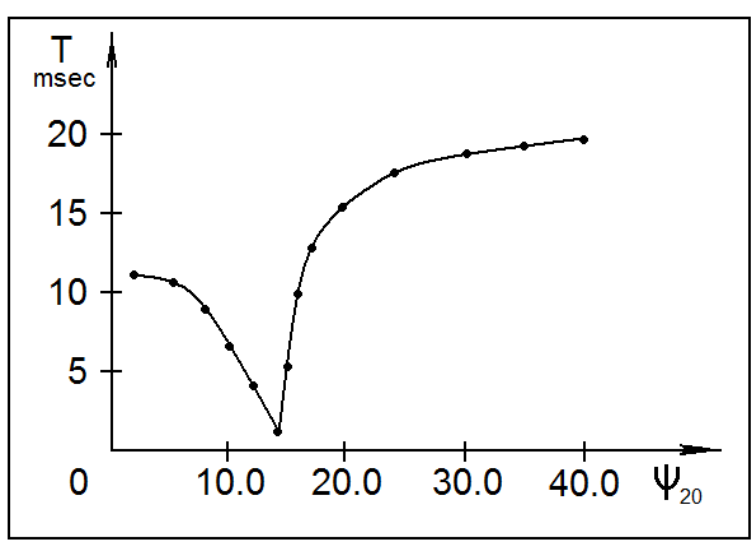

Fig. 4 CPU time as function of parameter $\psi_{20}$

The value $\psi_{20 \text { opt }}=14.35$ corresponds to the minimum CPU time $T_{\min }$ and integral $\mathrm{J}$, and is the same initial value of variable $\psi_{2}(t)$ which, according to the maximum principle, provides the maximum and constant value of a Hamiltonian of $\mathrm{H}$. The gain in time computed as time relation for TSO by the minimum time of $T_{\min }$ thus equal to 13.45 times. Dependences of the functions $\psi_{1}(t)$ and $\psi_{2}(t)$, and also a Hamiltonian of $H(t)$ are presented in Fig. 5.

Hamiltonian in this case is a constant and this fact corresponds to the maximum principle. It is interesting to analyze behavior of these functions with a non-optimal point of switching $t_{\mathrm{sw}}$ of the control function $u(t)$. Dependences of $u(t)$ in change of $t_{\mathrm{sw}}$ as parameter are presented in Fig. 6 and Fig. 7.

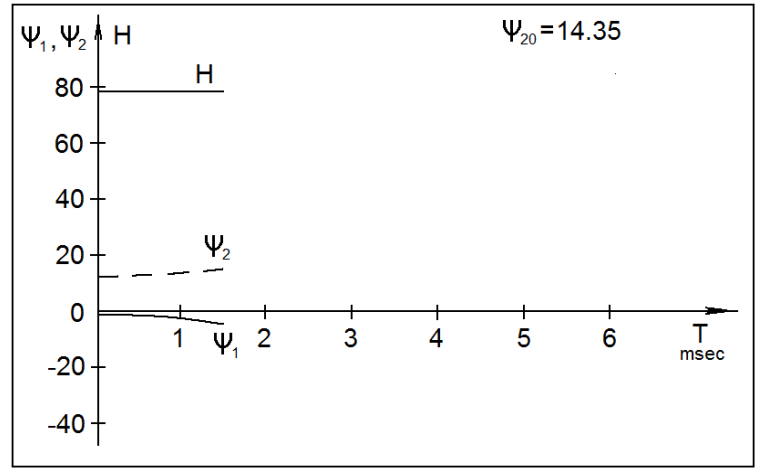

Fig. 5 dependencies of $\psi_{1}(t), \psi_{2}(t)$ and $H(t)$ for optimal $\psi_{20 o p t}$ 


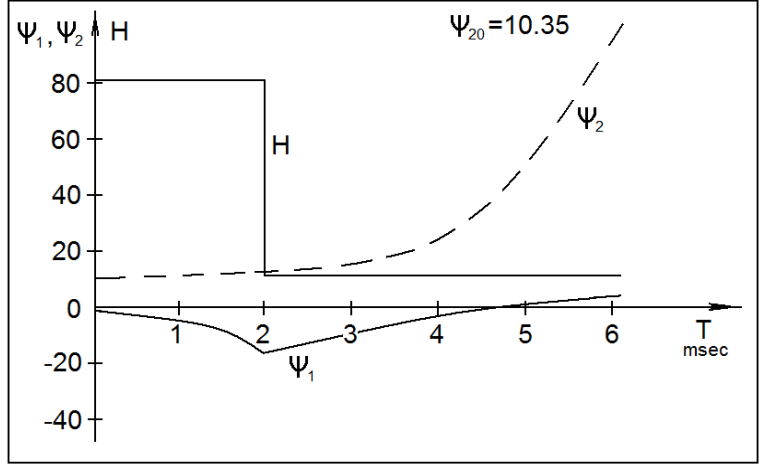

Fig. 6 dependencies of $\psi_{1}(t), \psi_{2}(t)$ and $H(t)$ for

$$
\psi_{20}<\psi_{20 o p t}
$$

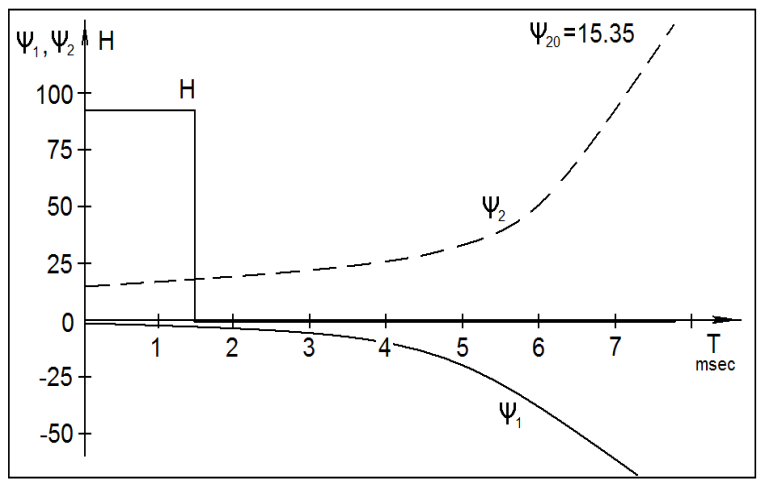

Fig. 7 dependencies of $\psi_{1}(t), \psi_{2}(t)$ and $H(t)$ for

$$
\psi_{20}>\psi_{20 o p t}
$$

Hamiltonian is changing in time when the switching point is differing from the optimal one. This criterion can be the basic in practical search of the optimal control function.

The analysis of optimization process of the presented circuit showed that application of the maximum principle really allows to find optimum structure of the control function $u(t)$, by means of iterative procedure. Criterion of the end of procedure is an invariable value of a Hamiltonian. Thus considerable reduction of CPU time in comparison with traditional approach is observed.

\section{Conclusion}

The task of constructing a time-minimized algorithm can be adequately solved on the basis of control theory. The design process in this case is formulated as a controlled dynamic system.

Analysis of the application of maximum principle to a problem of circuit optimization proves that the formerly studied effect of acceleration on the process of optimization appears owing to this principle. This means that the maximum principle of Pontryagin provides a theoretical justification for the acceleration effect that appears when we use the generalized formulation of process of circuit optimization. It is confirmed that the maximum principle allows for finding one or several local minima of the functional that is defined as the processor time. Aside from that, the use of the maximum principle provides the chance to significantly reduce the computing time for circuit optimization.

The analysis of optimization process of the presented circuit showed that application of the maximum principle really allows finding the optimum structure of the control vector $U$ by means of iterative procedure. The solution to this problem allows you to build an algorithm for optimizing the system in minimal time.

\section{References:}

[1] J.R. Bunch and D.J. Rose, (Eds.), Sparse Matrix Computations, Acad. Press, N.Y., 1976.

[2] O. Osterby and Z. Zlatev, Direct Methods for Sparse Matrices, Springer-Verlag, N.Y., 1983.

[3] N. Rabat, A.E. Ruehli, G.W. Mahoney and J.J. Coleman, A Survey of Macromodeling, Proc. of the IEEE Int. Symp. Circuits Systems, April, 1985, pp. 139-143.

[4] M. Tadeusiewicz, M. and A. Kuczynski, A very fast method for the DC analysis of diodetransistor circuits, Circuits Systems and Signal Processing, Vol. 32, No.3, 2013, pp. 433-451.

[5] R.K. Brayton, G.D. Hachtel and A.L. Sangiovanni-Vincentelli, A survey of optimization techniques for integrated-circuit design, Proceedings IEEE, Vol. 69, No. 10, 1981, pp. 1334-1362.

[6] A.E. Ruehli, (Ed.), Circuit analysis, simulation and design. part 2. Amsterdam: Elsevier Science Publishers, 1987.

[7] G. Stehr, M. Pronath, F. Schenkel, H. Graeb and K. Antreich, Initial sizing of analog integrated circuits by centering within topology-given implicit specifications, Proceedings of the IEEE/ACM International Conference on CAD, 2003, pp. 241-246.

[8] M. Hershenson, S. Boyd and T. Lee, Optimal design of a CMOS op-amp via geometric programming. IEEE Transactions on CAD of Integrated Circuits and Systems, Vol. 20, No. 1, 2001, pp. 1-21.

[9] I.S. Kashirskiy and I.K. Trokhimenko, General optimization for electronic circuits, Tekhnika, Kiev, 1979. 
[10] V. Rizzoli, A. Costanzo and C. Cecchetti, Numerical optimization of broadband nonlinear microwave circuits, IEEE MTT-S Int. Symp., Vol. 1, 1990, pp. 335-338.

[11] E.S. Ochotta, R.A.Rutenbar and L.R. Carley, Synthesis of High-Performance Analog Circuits in ASTRX/OBLX, IEEE Trans. on CAD, Vol.15, No. 3, 1996, pp. 273-294.

[12] A.M. Zemliak, Design of Analog Networks by Control Theory Methods, Part 1, Theory, Radioelectronics and Communications Systems, Vol. 47, No. 5, 2004, pp. 11-17.

[13] A.M. Zemliak, Analysis of Dynamic Characteristics of Process of Designing Analogue Circuits, Radioelectronics and Communications Systems, Vol. 50, No. 11, 2007, pp. 603-608.

[14] A. Zemliak and T. Markina, Behaviour of Lyapunov's function for different strategies of circuit optimization, International Journal of Electronics, Vol. 102, N 4, 2015, pp. 619-634.

[15] L.S. Pontryagin, V.G. Boltyanskii, R.V. Gamkrelidze and E.F. Mishchenko, The mathematical theory of optimal processes, Interscience Publishers, Inc., N.Y., 1962.

[16] L.W. Neustadt, Synthesis of time-optimal control systems. J. Math. Analysis and Appl., Vol. 1, 1960, pp. 484-492.

[17] J.B. Rosen, Iterative solution of nonlinear optimal control problems, J. SIAM, Control Series A, 1966, pp. 223-244.

[18] R.P. Fedorenko, Approximate Solution of Optimal Control Problems, Nauka, Moscow, 1978.

[19] L. Bourdin and E. Trélat, Pontryagin maximum principle for finite dimensional nonlinear optimal control problems on time scales. SIAM J. Control Optim., Vol 51, No. 5, 2013, pp. 3781-3813. 